\title{
HOMOTOPY ANALYSIS OF A FORCED NONLINEAR BEAM MODEL WITH QUADRATIC AND CUBIC NONLINEARITIES
}

\author{
Shahram Shahlaei-Far, Airton Nabarrete, José Manoel Balthazar \\ Aeronautics Institute of Technology, ITA, São José dos Campos, Brazil \\ e-mail: shazzz_85@yahoo.de
}

\begin{abstract}
This study investigates forced nonlinear vibrations of a simply supported Euler-Bernoulli beam on a nonlinear elastic foundation with quadratic and cubic nonlinearities. Applying the homotopy analysis method (HAM) to the spatially discretized governing equation, we derive novel analytical solutions and discuss their convergence to present nonlinear frequency responses with varying contributions of the nonlinearity coefficients. A comparison with numerical solutions is conducted and nonlinear time responses and phase planes are compared to the results from linear beam theory. The study demonstrates that apart from nonlinear problems of free vibrations, HAM is equally capable of solving strongly nonlinear problems of forced vibrations.
\end{abstract}

Keywords: forced nonlinear vibration, HAM, quadratic and cubic nonlinearities, Galerkin method

\section{Introduction}

Nonlinear vibrations of a simply supported Euler-Bernoulli beam on a nonlinear elastic foundation with distributed quadratic and cubic nonlinearities subject to harmonic excitation are, in nondimensional form, governed by (Abe, 2006)

$$
\frac{\partial^{2} w}{\partial t^{2}}+\frac{\partial^{4} w}{\partial x^{4}}+2 \mu \frac{\partial w}{\partial t}+\alpha_{2} w^{2}+\alpha_{3} w^{3}=F(x) \cos (\Omega t)
$$

subject to the boundary conditions

$$
w=\frac{\partial^{2} w}{\partial x^{2}}=0 \quad \text { at } \quad x=0,1
$$

where $w$ is the displacement of the beam, $\mu$ is the viscous damping coefficient, $\alpha_{2}$ and $\alpha_{3}$ are the quadratic and cubic nonlinearity coefficients, respectively, and $F(x)$ and $\Omega$ are the distribution and frequency of the harmonic load, respectively.

Abe (2006) provided numerical solutions for the cases of primary resonance and subharmonic resonance of the order one-half by means of the finite difference and shooting method, the first applied directly to Eqs. (1.1) and (1.2) and the latter to their spatially discretized form using the Galerkin method. He concluded that the Galerkin discretization yields more accurate results than the direct approach. Considering only small amplitude vibrations, he also obtained perturbative approximate solutions using the method of multiple scales (MMS) (Nayfeh and Mook, 1979) applied directly to Eq. (1.1) modeled as a weakly nonlinear system involving small perturbation parameters. However, Abe et al. $(1998, a, b, c, 2000)$ showed that for the direct approach it is unlikely to obtain highly accurate solutions as it is not possible to define a detuning parameter in the quadratic form between the natural and the excitation frequency.

The present work provides accurate analytical solutions to Eqs. (1.1) and (1.2) using the homotopy analysis method (HAM) introduced by Liao (Liao, 1992, 1995, 2003, 2004, 2009, 
2012; Liao and Cheung, 1998; Liao and Tan, 2007) to investigate the case when the excitation frequency is close to the natural frequency of the fundamental mode. Making no use of small or large parameters, this method allows us to consider the general nonlinear distributed-parameter system for small as well as large amplitude vibrations. Thus it overcomes the requirement for the MMS to model the problem as a weakly nonlinear system involving only small finite amplitudes. To ensure accurate results, we reduce the governing equation to an ordinary nonlinear differential equation with the Galerkin method before applying HAM. For the convergence analysis of the obtained analytical solutions, we plot the so-called $h$-curves for higher-order approximations and achieve the optimal value of $h$ by minimizing the square residual of the governing equation for a chosen order of the approximation. We verify our higher-order solutions by comparison with numerical solutions given by the fourth-order Runge-Kutta method. Demonstrating how powerful a first-order approximation of HAM already is, we derive explicit closed-form solutions of the mean of motion, amplitude and phase of the generalized coordinate to present frequency response curves for various values of the quadratic and cubic nonlinearity coefficients addressing the cases of softening- and hardening-type behavior. Moreover, we compare the nonlinear time responses and their respective phase planes for different values of the quadratic nonlinearities to the results from linear beam theory.

HAM has seen extensive successful applications to highly nonlinear problems in science and engineering (Wen and Cao, 2007; Hoseini et al., 2008; Pirbodaghi et al., 2009; Abbasbandy and Shivanian, 2011; Mastroberardino, 2011; Mehrizi et al., 2012; Mohammadpour et al., 2012; Mustafa et al., 2012; Sedighi et al., 2012; Ray and Sahoo, 2015). Previous applications of HAM have been mainly devoted to nonlinear differential equations of free vibratory continuous systems. As seen in Eq. (1.1), the important case of forced nonlinear vibrations of a damped system involving cubic and quadratic nonlinearities, where the mean of motion cannot be disregarded, poses a greater challenge with respect to finding analytical solutions and understanding nonlinear behavior. Our results provide an example, applicable to other beam models and boundary conditions, of a forced nonlinear vibratory system in which HAM yields accurate convergent solutions for all values of the relevant parameters.

\section{Governing differential equation}

The model is discretized by the Galerkin method with $w(x, t)=W(t) \phi(x)$, where $\phi(x)=\sqrt{2} \sin (\pi x)$ is the normalized eigenfunction of the fundamental mode and $W(t)$, the corresponding time-dependent amplitude, is the generalized coordinate. Thus, governing equation (1.1) is reduced to the nonlinear ordinary differential equation

$$
\ddot{W}+2 \mu \dot{W}+\omega^{2} W+\frac{8 \sqrt{2}}{3 \pi} \alpha_{2} W^{2}+\frac{3}{2} \alpha_{3} W^{3}=f \cos (\Omega t-\varphi)
$$

and without loss of generality subject to the initial conditions

$$
W(0)=\delta+A \quad \dot{W}(0)=0
$$

where $\omega=\pi^{2}$ is the normalized natural frequency of the fundamental mode, $f=\int_{0}^{1} F(x) \phi(x) d x$ is the first modal force, $A$ is an unknown amplitude and $\delta=(1 / T) \int_{0}^{T} W(t) d t$ is the mean of motion being generally nonzero for oscillations with the quadratic nonlinearity. The dot represents differentiation with respect to time $t$. Note that, for convenience and without loss of generality, we introduce the phase angle $\varphi$ in the expression of the harmonic load as a quantity to be determined.

Defining the variables

$$
\tau=\Omega t \quad W(t)=\delta+A V(\tau)
$$


and inserting them into Eqs. (2.1) and (2.2), we obtain

$$
\begin{aligned}
\Omega^{2} A & \frac{\partial^{2} V(\tau)}{\partial \tau^{2}}+\Omega 2 \mu A \frac{\partial V(\tau)}{\partial \tau}+\omega^{2}(\delta+A V(\tau))+\frac{8 \sqrt{2}}{3 \pi} \alpha_{2}(\delta+A V(\tau))^{2} \\
& +\frac{3}{2} \alpha_{3}(\delta+A V(\tau))^{3}=f_{1} \cos \tau+f_{2} \sin \tau
\end{aligned}
$$

subject to the initial conditions

$$
V(0)=1 \quad \frac{\partial V(0)}{\partial \tau}=0
$$

with the constants $f_{1}$ and $f_{2}$ satisfying

$$
f_{1}^{2}+f_{2}^{2}=f^{2} \quad \varphi=\arctan \frac{f_{2}}{f_{1}}
$$

\section{Homotopy analysis method}

The homotopy analysis method is a nonperturbative analytical technique for solving nonlinear differential equations. By means of an embedding parameter ranging from zero to one, it transforms a nonlinear differential equation into an infinite number of linear differential equations and derives a family of solution series.

The periodic solution to Eq. (2.4) can be expressed by a set of base functions

$$
\{\sin (m \tau), \cos (m \tau) \mid m=1,2,3, \ldots\}
$$

such that

$$
V(\tau)=\sum_{k=1}^{i n f t y}\left(\alpha_{k} \sin (k \tau)+\beta_{k} \cos (k \tau)\right)
$$

where $\alpha_{k}$ and $\beta_{k}$ are coefficients to be determined. We choose the initial guess as

$$
V_{0}=\cos \tau
$$

which satisfies the initial conditions of Eq. (2.5). To ensure the rule of solution expression given by Eq. (3.2), we choose the linear operator to be

$$
\mathcal{L}[\phi(\tau, q)]=\Omega^{2}\left(\frac{\partial^{2} \phi(\tau, q)}{\partial \tau^{2}}+\phi(\tau, q)\right)
$$

with the property

$$
\mathcal{L}\left[C_{1} \sin \tau+C_{2} \cos \tau\right]=0
$$

where $C_{1}$ and $C_{2}$ are constants of integration. According to Eq. (2.4), we define the nonlinear operator

$$
\begin{aligned}
\mathcal{N}[\phi & (\tau, q), \Lambda(q), \Delta(q)]=\Omega^{2} \Lambda(q) \frac{\partial^{2} \phi(\tau, q)}{\partial \tau^{2}}+2 \mu \Omega \Lambda(q) \frac{\partial \phi(\tau, q)}{\partial \tau} \\
& +\omega^{2}(\Delta(q)+\Lambda(q) \phi(\tau, q))+\frac{8 \sqrt{2}}{3 \pi} \alpha_{2}(\Delta(q)+\Lambda(q) \phi(\tau, q))^{2} \\
& +\frac{3}{2} \alpha_{3}(\Delta(q)+\Lambda(q) \phi(\tau, q))^{3}-f_{1} \cos \tau-f_{2} \sin \tau
\end{aligned}
$$


where $q \in[0,1]$ is the embedding parameter, $\phi(\tau, q)$ is a function of $\tau$ and $q, \Lambda(q)$ and $\Delta(q)$ are functions of $q$. The zeroth-order deformation equation is given by

$$
(1-q) \mathcal{L}\left[\phi(\tau, q)-V_{0}(\tau)\right]=q h H(\tau) \mathcal{N}[\phi(\tau, q), \Lambda(q), \Delta(q)]
$$

where $h \neq 0$ is the convergence-control parameter and $H(\tau)$ a nonzero auxiliary function. For $q=0$ and $q=1$ we have

$$
\begin{array}{lll}
\phi(\tau, 0)=V_{0}(\tau) & \phi(\tau, 1)=V(\tau) & \Lambda(0)=A_{0} \\
\Lambda(1)=A & \Delta(0)=\delta_{0} & \Delta(1)=\delta
\end{array}
$$

Thus, the function $\phi(\tau, q)$ varies from the initial guess $V_{0}(\tau)$ to the desired solution as $q$ varies from 0 to 1. The Taylor expansions of $\phi(\tau, q), \Lambda(q)$ and $\Delta(q)$ with respect to $q$ are

$$
\begin{array}{ll}
\phi(\tau, q) & =V_{0}(\tau)+\sum_{m=1}^{\text {infty }} V_{m}(\tau) q^{m} \quad \Lambda(q)=A_{0}+\sum_{m=1}^{\text {infty }} A_{m} q^{m} \\
\Delta(q) & =\delta_{0}+\sum_{m=1}^{\text {infty }} \delta_{m} q^{m}
\end{array}
$$

where

$$
V_{m}(\tau)=\left.\frac{1}{m !} \frac{\partial^{m} \phi(\tau, q)}{\partial q^{m}}\right|_{q=0} \quad A_{m}=\left.\frac{1}{m !} \frac{\partial^{m} \Lambda(q)}{\partial q^{m}}\right|_{q=0} \quad \delta_{m}=\left.\frac{1}{m !} \frac{\partial^{m} \Delta(q)}{\partial q^{m}}\right|_{q=0}
$$

Choosing properly the auxiliary function $H(\tau)$ and the convergence-control parameter $h$, the series in Eqs. (3.9) converge when $q=1$, such that

$$
V(\tau)=V_{0}(\tau)+\sum_{m=1}^{\text {infty }} V_{m}(\tau) \quad A=A_{0}+\sum_{m=1}^{\text {infty }} A_{m} \quad \delta=\delta_{0}+\sum_{m=1}^{\infty} \delta_{m}
$$

Differentiating zeroth-order equation (3.7) $m$ times with respect to $q$, dividing it by $m$ ! and setting $q=0$, the $m$-th order deformation equation is obtained as

$$
\mathcal{L}\left[V_{m}(\tau)-\chi_{m} V_{m-1}(\tau)\right]=h H(\tau) R_{m}\left(\mathbf{V}_{m-1}, \mathbf{A}_{m-1}, \boldsymbol{\delta}_{m-1}\right)
$$

subject to the initial conditions

$$
V_{m}(0)=\frac{\partial V_{m}(0)}{\partial \tau}=0
$$

where

$$
\begin{array}{ll}
\chi_{m}= \begin{cases}0 & m \leqslant 1 \\
1 & m>1\end{cases} & \mathbf{V}_{m-1}=\left[V_{0}(\tau), V_{1}(\tau), \ldots, V_{m-1}(\tau)\right] \\
\mathbf{A}_{m-1}=\left[A_{0}, A_{1}, \ldots, A_{m-1}\right] & \boldsymbol{\delta}_{m-1}=\left[\delta_{0}, \delta_{1}, \ldots, \delta_{m-1}\right]
\end{array}
$$


and

$$
\begin{aligned}
R_{m} & \left(\mathbf{V}_{m-1}, \mathbf{A}_{m-1}, \boldsymbol{\delta}_{m-1}\right)=\left.\frac{1}{(m-1) !} \frac{d^{m-1} \mathcal{N}[\phi(\tau, q), \Lambda(q), \Delta(q)]}{d q^{m-1}}\right|_{q=0} \\
& =\Omega^{2} A_{m-1} \frac{\partial^{2} V_{m-1}}{\partial \tau^{2}}+2 \mu \Omega A_{m-1} \frac{\partial V_{m-1}}{\partial \tau}+\omega^{2}\left(\delta_{m-1}+A_{m-1} V_{m-1}\right) \\
& +\frac{8 \sqrt{2}}{3 \pi} \alpha_{2} \sum_{n=0}^{m-1}\left(\delta_{n}+A_{n} V_{n}\right)\left(\delta_{m-1-n}+A_{m-1-n} V_{m-1-n}\right) \\
& +\frac{3}{2} \alpha_{3} \sum_{n=0}^{m-1}\left(\sum_{j=0}^{n}\left(\delta_{j}+A_{j} V_{j}\right)\left(\delta_{n-j}+A_{n-j} V_{n-j}\right)\right) \\
& \cdot\left(\delta_{m-1-n}+A_{m-1-n} V_{m-1-n}\right)-\left(1-\chi_{m}\right)\left(f_{1} \cos \tau+f_{2} \sin \tau\right) \\
& =C_{m, 0}+\sum_{k=1}^{l(m)}\left(c_{m, k}\left(\mathbf{V}_{m-1}, \mathbf{A}_{m-1}, \boldsymbol{\delta}_{m-1}\right) \cos (k \tau)+d_{m, k}\left(\mathbf{V}_{m-1}, \mathbf{A}_{m-1}, \boldsymbol{\delta}_{m-1}\right) \sin (k \tau)\right)
\end{aligned}
$$

For the nonzero auxiliary function to obey the rule of solution expression and the rule of coefficient ergodicity, we choose it to be

$$
H(\tau)=\cos (2 \tau)
$$

where $\kappa$ is an integer. It can be determined uniquely as $H(\tau)=1$.

According to the property of the linear operator, if the terms $\sin \tau$ and $\cos \tau$ exist in $R_{m}$, the secular terms $\tau \cos \tau$ and $\tau \sin \tau$ will appear in the final solution, therefore $c_{m, 1}$ and $d_{m, 1}$ have to be equal to zero. Moreover, if $C_{m, 0} \neq 0$, a constant term will appear in the final solution violating the rule of solution expression, thus it must be set to zero.

The general solution to Eq. (3.12) for $m \geqslant 1$ is derived to be

$$
V_{m}(\tau)=\chi_{m} V_{m-1}(\tau)+\frac{h}{\Omega^{2}} \sum_{k=2}^{l(m)} \frac{c_{m, k} \cos (k \tau)+d_{m, k} \sin (k \tau)}{1-k^{2}}+C_{1} \sin \tau+C_{2} \cos \tau
$$

where $C_{1}$ and $C_{2}$ need to be determined by the initial conditions in Eq. (3.13).

For the first-order approximation $(\mathrm{m}=1)$ we obtain from Eq. (3.15)

$$
\begin{aligned}
& R_{1}\left(\mathbf{V}_{0}, \mathbf{A}_{0}, \boldsymbol{\delta}_{0}\right)=-\Omega^{2} A_{0} \cos \tau-2 \mu \Omega A_{0} \sin \tau+\omega^{2}\left(\delta_{0}+A_{0} \cos \tau\right) \\
& +\frac{8 \sqrt{2}}{3 \pi} \alpha_{2}\left(\delta_{0}+A_{0} \cos \tau\right)^{2}+\frac{3}{2} \alpha_{3}\left(\delta_{0}+A_{0} \cos \tau\right)^{3}-f_{1} \cos \tau \\
& \quad-f_{2} \sin \tau=\frac{8 \sqrt{2}}{6 \pi} \alpha_{2} A_{0}^{2}+\left(\omega^{2}+\frac{9}{4} \alpha_{3}\right) \delta_{0}+\frac{8 \sqrt{2}}{3 \pi} \alpha_{2} \delta_{0}^{2}+\frac{3}{2} \alpha_{3} \delta_{0}^{3} \\
& +\left(-\Omega^{2} A_{0}+\omega^{2} A_{0}+\frac{16 \sqrt{2}}{3 \pi} \alpha_{2} \delta_{0} A_{0}+\frac{9}{2} \alpha_{3} \delta_{0}^{2} A_{0}+\frac{9}{8} \alpha_{3} A_{0}^{3}-f_{1}\right) \cos \tau \\
& +\left(-2 \mu \Omega A_{0}-f_{2}\right) \sin \tau+\left(\frac{8 \sqrt{2}}{6 \pi} \alpha_{2} A_{0}^{2}+\frac{9}{8} \alpha_{3} \delta_{0} A_{0}^{2}\right) \cos (2 \tau)+\frac{3}{8} \alpha_{3} A_{0}^{3} \cos (3 \tau) \\
& =C_{1,0}+\sum_{k=1}^{l(1)=3}\left(c_{1, k}\left(\mathbf{V}_{0}, \mathbf{A}_{0}, \boldsymbol{\delta}_{0}\right) \cos (k \tau)+d_{1, k}\left(\mathbf{V}_{0}, \mathbf{A}_{0}, \boldsymbol{\delta}_{0}\right) \sin (k \tau)\right)
\end{aligned}
$$

with $d_{1,2}=d_{1,3}=0$. Enforcing the constant term and the coefficients of $\sin \tau$ and $\cos \tau$ to be equal to zero, and using the conditions in Eq. (2.6), we obtain steady state solutions of the mean of motion $\delta_{0}$, the amplitude $A_{0}$ and the phase $\varphi$, respectively, as

$$
\delta_{0}=-\frac{16 \sqrt{2} \alpha_{2}}{27 \alpha_{3} \pi}-\frac{K_{1}}{27 \alpha_{3} \sqrt[3]{2} \pi \sqrt[3]{K_{2}+\sqrt{K_{2}^{2}+4 K_{1}^{3}}}}+\frac{1}{54 \alpha_{3} \sqrt[3]{2} \pi} \sqrt[3]{K_{2}+\sqrt{K_{2}^{2}+4 K_{1}^{3}}}
$$


with

$$
K_{1}=-2048 \alpha_{2}^{2}+162 \alpha_{3} \pi\left(9 A_{0}^{2} \alpha_{3} \pi+4 \pi^{5}\right) \quad K_{2}=-131072 \sqrt{2} \alpha_{2}^{3}+62208 \sqrt{2} \alpha_{2} \alpha_{3} \pi^{6}
$$

and

$$
\begin{aligned}
& \left(\left(\omega^{2}-\Omega^{2}\right) A_{0}+\frac{16 \sqrt{2}}{3 \pi} \alpha_{2} \delta_{0} A_{0}+\frac{9}{2} \alpha_{3} \delta_{0}^{2} A_{0}+\frac{9}{8} \alpha_{3} A_{0}^{3}\right)^{2}+\left(-2 \mu \Omega A_{0}\right)^{2}=f^{2} \\
& \varphi=\arctan \frac{2 \mu \Omega}{\Omega^{2}-\omega^{2}-\frac{16 \sqrt{2}}{3 \pi} \alpha_{2} \delta_{0}-\frac{9}{2} \alpha_{3} \delta_{0}^{2}-\frac{9}{8} \alpha_{3} B} \\
& B=\frac{\omega^{2} \delta_{0}+\frac{8 \sqrt{2}}{3 \pi} \alpha_{2} \delta_{0}^{2}+\frac{3}{2} \alpha_{3} \delta_{0}^{3}}{\frac{9}{4} \alpha_{3} \delta_{0}+\frac{8 \sqrt{2}}{6 \pi} \alpha_{2}}
\end{aligned}
$$

Finally, solving the first-order deformation equation of Eq. (3.12), the general solution is

$$
\begin{aligned}
V_{1}(\tau) & =\frac{h}{\Omega^{2}} \sum_{k=2}^{l(1)=3} \frac{c_{1, k}}{1-k^{2}} \cos (k \tau)+C_{1} \sin \tau+C_{2} \cos \tau \\
= & \frac{h}{\Omega^{2}}\left(\left(\frac{4 \sqrt{2}}{9 \pi} \alpha_{2}+\frac{3}{4} \alpha_{3} \delta_{0}\right) A_{0}^{2}(\cos \tau-\cos (2 \tau))+\frac{3}{64} \alpha_{3} A_{0}^{3}(\cos \tau-\cos (3 \tau))\right)
\end{aligned}
$$

where the constants $C_{1}$ and $C_{2}$ are obtained from the initial conditions in Eq. (3.13).

Thus, with Eqs. (3.11), the first-order approximation of $W(t)$ becomes

$$
\begin{aligned}
W(t) & =\delta+A V(\tau) \approx \delta_{0}+A_{0}\left(V_{0}(\tau)+V_{1}(\tau)\right) \\
= & \delta_{0}+\frac{h}{\Omega^{2}}\left(\left(\frac{\Omega^{2}}{h} A_{0}+\frac{4 \sqrt{2}}{9 \pi} \alpha_{2} A_{0}^{3}+\frac{3}{4} \alpha_{3} \delta_{0} A_{0}^{3}+\frac{3}{64} \alpha_{3} A_{0}^{4}\right) \cos (\Omega t)\right. \\
+ & \left.\left(\frac{4 \sqrt{2}}{9 \pi} \alpha_{2} A_{0}^{3}+\frac{3}{4} \alpha_{3} \delta_{0} A_{0}^{3}\right) \cos (2 \Omega t)+\frac{3}{64} \alpha_{3} A_{0}^{4} \cos (3 \Omega t)\right)
\end{aligned}
$$

\section{Convergence of HAM solution}

Applying HAM to a nonlinear problem results in a family of solution series which depend on the convergence-control parameter $h$. In order to ensure the convergence and rate of the approximation for the HAM solution, valid convergence regions for the auxiliary parameter $h$ need to be obtained. By means of plotting $h$-curves this can be achieved and thus a convergent solution series is guaranteed. Since a valid region comprises a range of possible values of $h$, the optimal choice is obtained by minimizing the square residual of the governing equation for a given order of the approximation. For this, we consider the Nth-order approximations of Eqs. (3.11) given by

$$
V_{N}(\tau)=V_{0}(\tau)+\sum_{m=1}^{N} V_{m}(\tau) \quad A=A_{0}+\sum_{m=1}^{N} A_{m} \quad \delta=\delta_{0}+\sum_{m=1}^{N} \delta_{m}
$$

Inserting Eqs. (4.1)-(4.3) into Eq. (3.6) with $q=1$, we can define the square residual error for the $N$-th order approximation as

$$
e_{N}(h)=\int_{0}^{1}\left(\mathcal{N}\left[V_{N}(\tau), A_{N}, \delta_{N}\right]\right)^{2} d \tau
$$


The solution series is convergent when $e_{N}(h) \rightarrow 0$ as $N \rightarrow \infty$. The optimal value of $h$ for a given order $N$ of the approximation is obtained by the solution of the algebraic equation

$$
\frac{d e_{N}}{d h}=0
$$

It is to be noted that the calculation for each order is done separately and not iteratively.

(a)

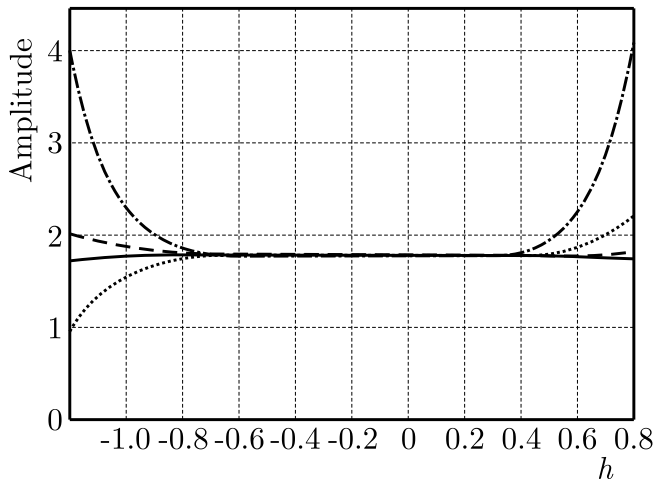

(c)

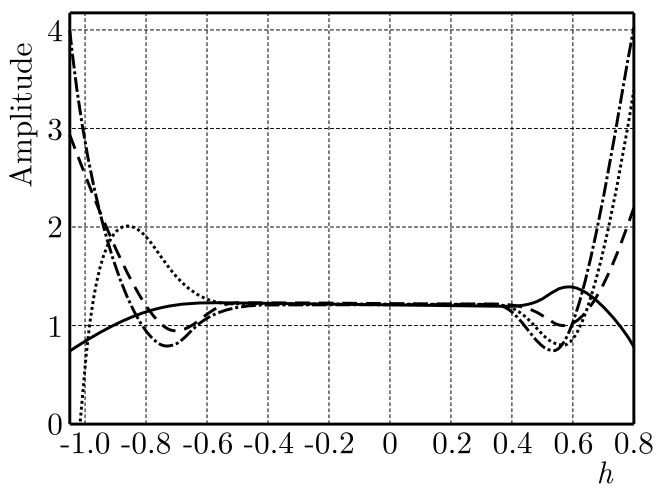

(b)

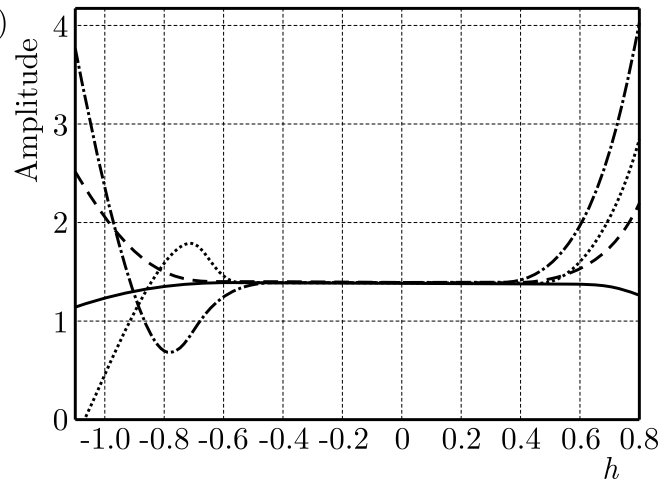

$$
\begin{aligned}
& \text { - } 2^{\text {nd }} \text { order } \\
& --4^{\text {th }} \text { order } \\
& \cdots \cdots \cdots \cdots \cdot 6^{\text {th }} \text { order } \\
& \text {-..- } 8^{\text {th }} \text { order }
\end{aligned}
$$

Fig. 1 . $h$-curves of the amplitude $A$ for $\alpha_{2}=5$ : (a) $\alpha_{3}=0.5$, (b) $\alpha_{3}=1$, (c) $\alpha_{3}=1.5$

Figure 1 shows the effect of the auxiliary parameter on the solution convergence for higher-order approximations. The valid region is characterized by the flat portion which is common to all $h$-curves displayed. The corresponding optimal values of $h$ for varying values of the cubic nonlinearity coefficient $\alpha_{3}$ are presented in Table 1 using the software Mathematica.

\section{Discussion of results}

A simply supported Euler-Bernoulli beam resting on a nonlinear elastic foundation with quadratic and cubic nonlinearities is considered. First, higher-order approximations from the general solution in Eq. (3.17), obtained with the software Mathematica, are compared to numerical results. Secondly, considering the first-order approximation of HAM, the frequency response curves of the amplitude obtained in Eq. (3.20) $)_{1}$ are presented for different values of the quadratic and cubic nonlinearity coefficients. Moreover, the nonlinear time response curves and phase planes are compared to the results from linear beam theory showing the effect of these nonlinearities upon the distributed-parameter system.

In Fig. 2, the accuracy of nonlinear time responses obtained by a sixth-order HAM approximation for $\alpha_{2}=5$ is validated by comparison with the numerical results achieved by the fourth-order Runge-Kutta method for varying values of $\alpha_{3}$ and the corresponding optimal values of the auxiliary parameter $h$. There is accurate agreement between the analytical and numerical results. 
Table 1. Optimal values of $h$ and minimum values of $e_{N}$ for $\mu=0.05, F(x)=\sqrt{2} \sin (\pi x)$, $\alpha_{2}=5$

\begin{tabular}{|c|c|c|c|}
\hline$\alpha_{3}$ & $N$ & Optimal value of $h$ & Minimum value of $e_{N}$ \\
\hline \hline \multirow{4}{*}{0.5} & 2 & -0.3567 & $8.374 \cdot 10^{-4}$ \\
\cline { 2 - 4 } & 4 & -0.3324 & $1.705 \cdot 10^{-7}$ \\
\cline { 2 - 4 } & 6 & -0.3286 & $4.119 \cdot 10^{-9}$ \\
\cline { 2 - 4 } & 8 & -0.3045 & $7.805 \cdot 10^{-11}$ \\
\hline \multirow{4}{*}{1} & 2 & -0.2943 & $5.096 \cdot 10^{-2}$ \\
\cline { 2 - 4 } & 4 & -0.2755 & $1.643 \cdot 10^{-5}$ \\
\cline { 2 - 4 } & 6 & -0.2619 & $4.295 \cdot 10^{-6}$ \\
\cline { 2 - 4 } & 8 & -0.2578 & $9.349 \cdot 10^{-8}$ \\
\hline 1.5 & 2 & -0.2581 & $6.912 \cdot 10^{-1}$ \\
\cline { 2 - 4 } & 4 & -0.2319 & $3.782 \cdot 10^{-2}$ \\
\cline { 2 - 4 } & 6 & -0.2247 & $2.186 \cdot 10^{-4}$ \\
\cline { 2 - 4 } & 8 & -0.2169 & $6.237 \cdot 10^{-6}$ \\
\hline
\end{tabular}

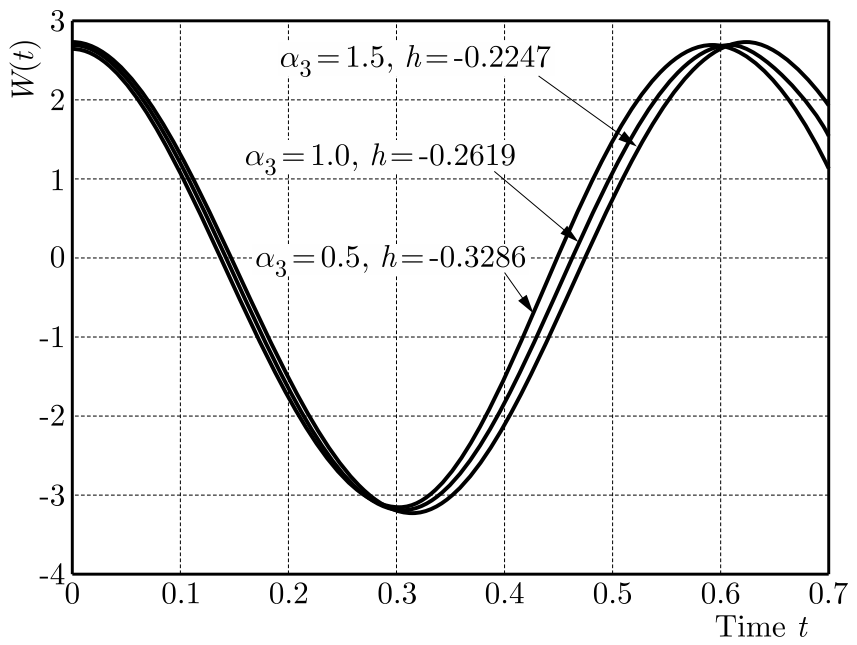

Fig. 2. Comparison of sixth-order HAM solutions (solid line) with numerical results by the fourth-order Runge-Kutta method (symbols)

Fixing $\alpha_{2}=5$ and $\alpha_{3}=0.5$ and using the optimal values of $h$ from Table 1, approximations of order 2, 4, 6 and 8 are compared to numerical results (fourth-order Runge-Kutta) in Table 2. The results show that low-order approximations by HAM agree well with numerical solutions, although by increasing the order of HAM iterations, the accuracy increases.

For the rest of this Section, we consider the first-order approximation by HAM. In Fig. 3, we investigate the influence of the nonlinearity coefficients on the frequency response curves, that is, we show the variation of the response curves with $\alpha_{2}$ for different values of $\alpha_{3}$. In Fig. 3a, as the excitation frequency $\Omega$ nears the fundamental frequency $\omega$, the response of the system exhibits hardening-spring nonlinear characteristics due to the cubic nonlinearity. Increasing the value of $\alpha_{3}$, this hardening-type behavior is further increased. In the presence of both $\alpha_{2}$ and $\alpha_{3}$, in Fig. 3b, the response is almost linear (for $\alpha_{3}=0.5$ ) suggesting that the magnitude of the nonlinearities cancel each other out. Further increase of $\alpha_{3}$ results, as in the previous case, in hardening-type behavior. Figure 3c predicts softening- as well as hardening-type responses for increasing values of $\alpha_{3}$ demonstrating the softening effect of the quadratic nonlinearity. This transition is also evident in Fig. 3d, whereby the softening-spring effect is more pronounced. For higher values of the quadratic nonlinearity, the softening effect on the system becomes more distinctive. 
Table 2. Comparison of higher-order HAM solutions with numerical results for $\mu=0.05$, $F(x)=\sqrt{2} \sin (\pi x), \alpha_{2}=5, \alpha_{3}=0.5$

\begin{tabular}{|c|c|c|c|c|c|}
\hline \multirow{2}{*}{$\begin{array}{c}\text { Time } \\
t\end{array}$} & \multicolumn{4}{|c|}{ HAM solution } & \multirow{2}{*}{$\begin{array}{c}\text { Numerical } \\
\text { results }\end{array}$} \\
\hline & 2 -nd order & 4 -th order & 6 -th order & 8-th order & \\
\hline$\overline{0}$ & 2.70874 & 2.71538 & 2.71796 & 2.71923 & 2.71925 \\
\hline 0.1 & 1.34795 & 1.35269 & 1.35344 & 1.35416 & 1.35418 \\
\hline 0.2 & -1.50326 & -1.51083 & -1.51278 & -1.51490 & -1.51492 \\
\hline 0.3 & -3.21418 & -3.21964 & -3.22461 & -3.22791 & -3.22793 \\
\hline$\overline{0.4}$ & -2.15817 & -2.16227 & -2.16435 & -2.16520 & -2.16521 \\
\hline 0.5 & 0.65104 & 0.66129 & 0.66483 & 0.66659 & 0.66662 \\
\hline 0.6 & 2.61598 & 2.62004 & 2.62135 & 2.62337 & 2.62339 \\
\hline 0.7 & 1.92956 & 1.93118 & 1.93207 & 1.93479 & 1.93480 \\
\hline
\end{tabular}

(a)

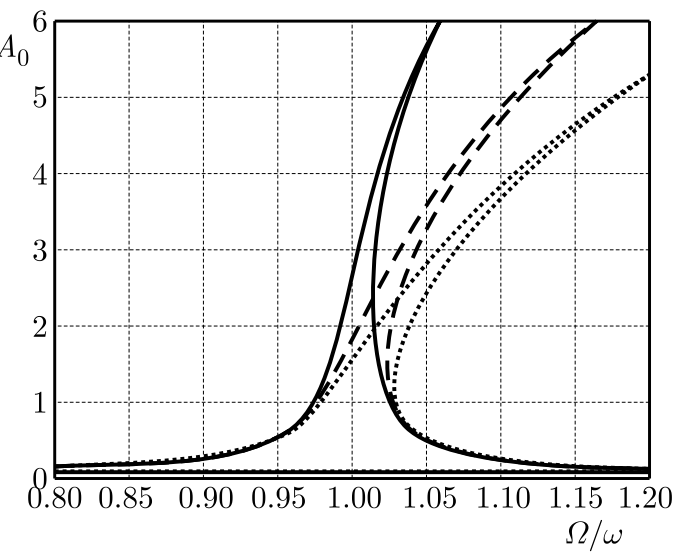

(b)

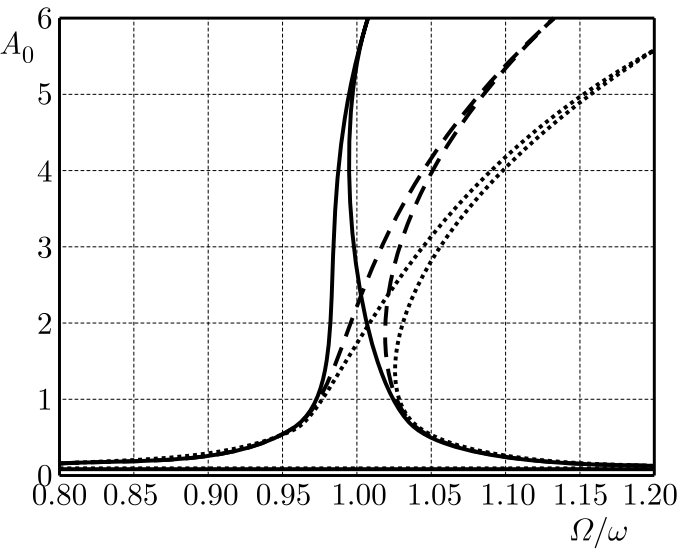

(c)

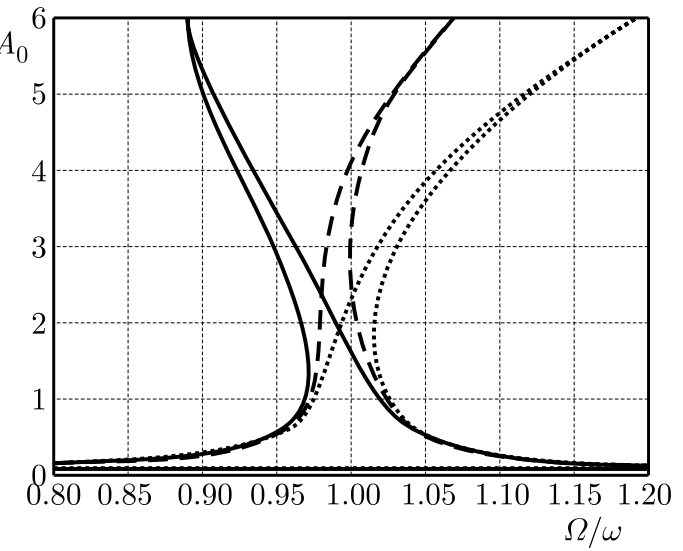

(d)

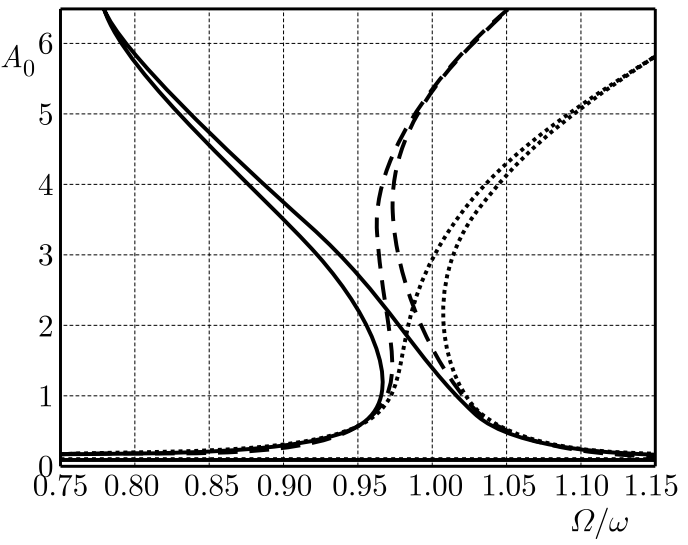

Fig. 3. Amplitude-frequency curves for $\mu=0.05, F(x)=\sqrt{2} \sin (\pi x), \alpha_{3}=0.5$ (solid line), $\alpha_{3}=1$ (dashed line), $\alpha_{3}=1.5$ (dotted line): (a) $\alpha_{2}=5$, (b) $\alpha_{2}=7.4$, (c) $\alpha_{2}=10.5$, (d) $\alpha_{2}=12$

Investigating the impact of the quadratic nonlinearity on the distributed-parameter system we compare, in Fig. 4, the nonlinear time responses obtained by HAM with those from linear beam theory maintaining a fixed value for $\alpha_{3}$ and varying values of the quadratic nonlinearity coefficient $\alpha_{2}$. The optimal value of $h$ is obtained by solving Eq. (4.5) for $N=1$. It is evident that for lower values of $\alpha_{2}$ there is more agreement between the linear and nonlinear time response, whereas by increasing the value of $\alpha_{2}$, the difference becomes considerable.

In Fig. 5, the phase planes for both nonlinear and linear responses are presented for different values of $\alpha_{2}$ while fixing $\alpha_{3}=0.5$. With an increase in $\alpha_{2}$, the phase planes significantly diverge from their linear counterparts emphasizing the effect of the quadratic nonlinearity term on 
(a)

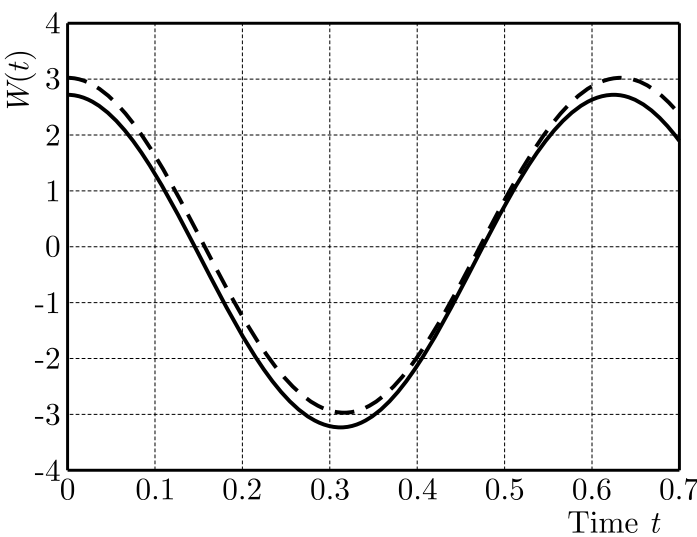

(c)

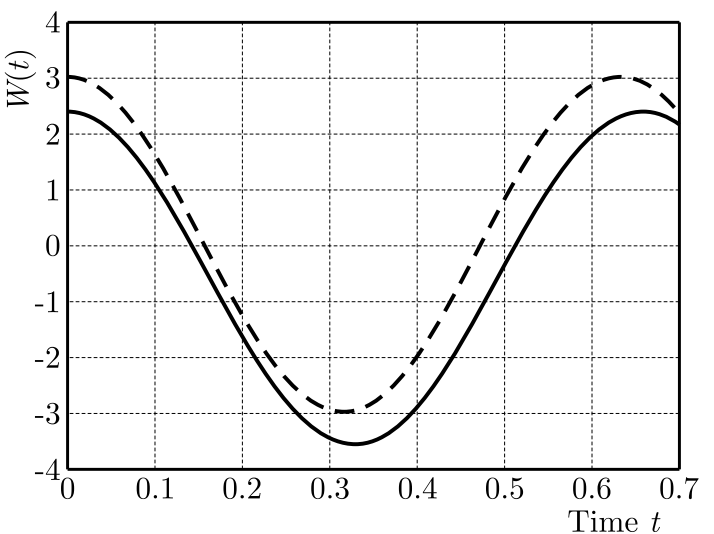

(b)
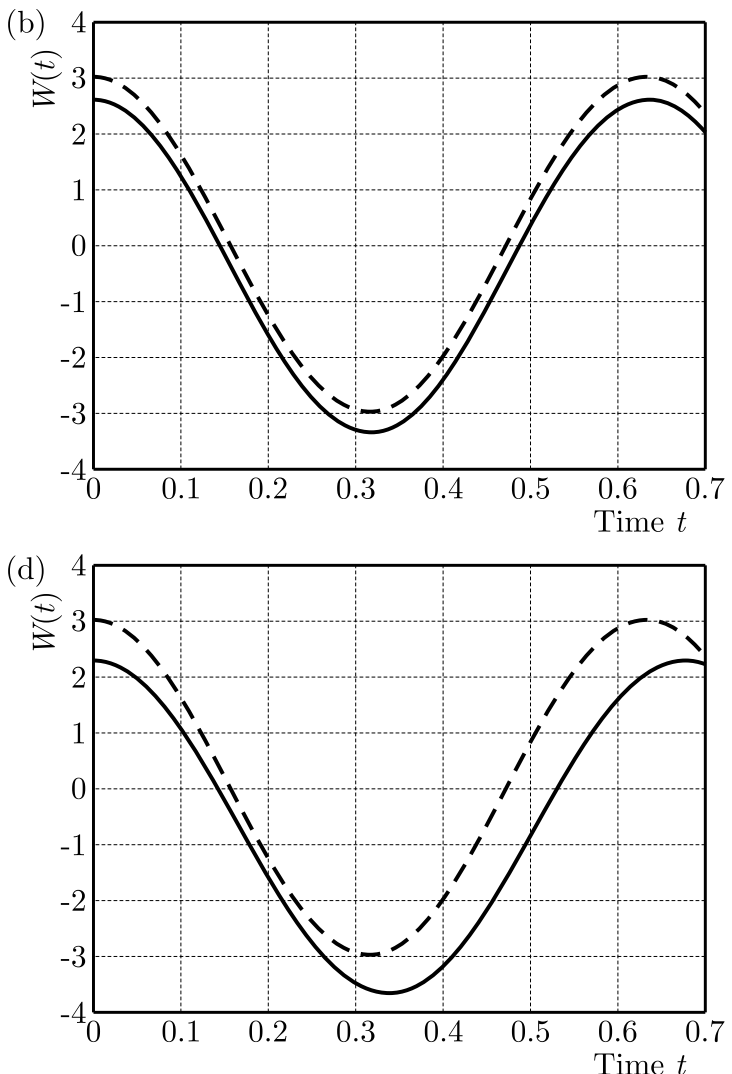

Fig. 4. Linear time response (dashed line) versus nonlinear time response (solid line) for $\mu=0.05$, $F(x)=\sqrt{2} \sin (\pi x), \alpha_{3}=0.5, h=-0.3719:$ (a) $\alpha_{2}=5(\Omega / \omega=0.9806)$, (b) $\alpha_{2}=7.4(\Omega / \omega=0.9910)$, (c) $\alpha_{2}=10.5(\Omega / \omega=1.0075),(\mathrm{d}) \alpha_{2}=12(\Omega / \omega=1.0160)$
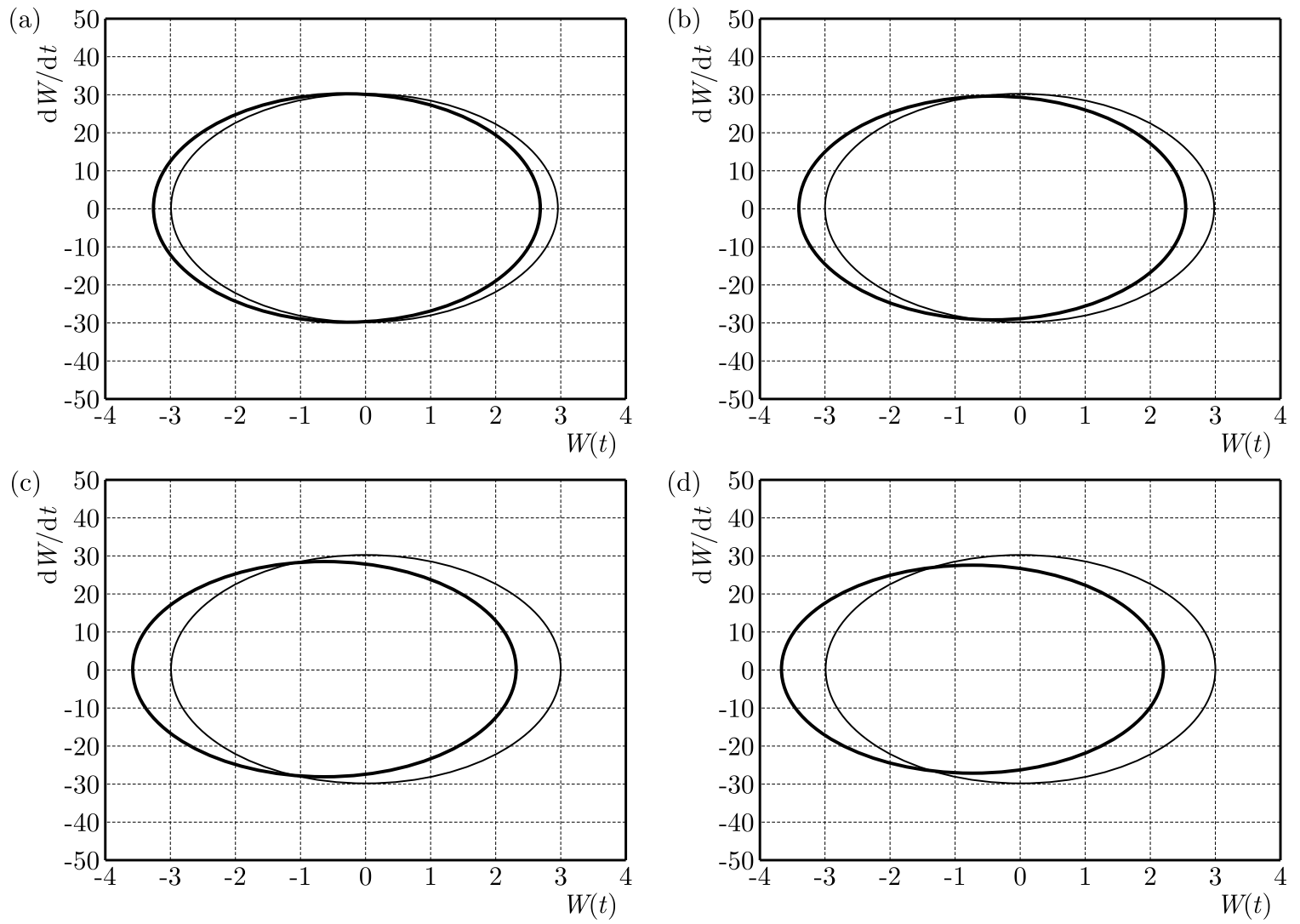

Fig. 5. Phase plane of linear response (thin line) versus nonlinear response (thick line) for $\mu=0.05$, $F(x)=\sqrt{2} \sin (\pi x), \alpha_{3}=0.5, h=-0.3719:$ (a) $\alpha_{2}=5(\Omega / \omega=0.9806)$, (b) $\alpha_{2}=7.4(\Omega / \omega=0.9910)$, (c) $\alpha_{2}=10.5(\Omega / \omega=1.0075),(d) \alpha_{2}=12(\Omega / \omega=1.0160)$ 
the system when the excitation frequency is close to the natural frequency of the fundamental mode.

It should be noted that the analysis presented in this study can be expanded to predict beam responses for different boundary conditions. To this end, the mode shape $\phi(x)$ satisfying the boundary conditions at both ends should be inserted into $w(x, t)=W(t) \phi(x)$ for the spatial discretization by the Galerkin method. The solutions can then be derived analogously with the methodology described in Section 3.

\section{Conclusion}

The present study provides analytical solutions to forced nonlinear vibrations of a simply supported Euler-Bernoulli beam resting on a nonlinear elastic foundation with quadratic and cubic nonlinearities using the homotopy analysis method. The convergence of the solution has been investigated by optimizing the value of the auxiliary convergence-control parameter $h$. Using the optimal values of $h$, higher-order solutions by HAM have been compared to numerical results demonstrating the effectiveness of the method for low-order approximations and varying values of the cubic nonlinearity. The derived closed-form solution of the amplitude yields frequency response curves for various values of the quadratic and cubic nonlinearity coefficients presenting their softening-/hardening-type effect on the distributed-parameter system. Phase planes and nonlinear time response curves illustrate the considerable difference with respect to the results from linear beam theory for various values of the quadratic nonlinearity coefficient. The findings reveal that HAM is a general solution method that can successfully address highly nonlinear problems of forced vibrations.

\section{Acknowledgement}

The authors acknowledge the financial support received during this work from CAPES and the $\mathrm{MCT} / \mathrm{CNPq} / \mathrm{FAPEMIG-National} \mathrm{Institute} \mathrm{of} \mathrm{Science} \mathrm{and} \mathrm{Technology} \mathrm{on} \mathrm{Smart} \mathrm{Structures} \mathrm{in} \mathrm{Engine-}$ ering, grant number 574001/2008-5.

\section{References}

1. Abbasbandy S., Shivanian E., 2011, Predictor homotopy analysis method and its application to some nonlinear problems, Communications in Nonlinear Science and Numerical Simulation, 16, $2456-2468$

2. ABE A., 2006, On non-linear vibration analyses of continuous systems with quadratic and cubic non-linearities, International Journal of Non-Linear Mechanics, 41, 873-879

3. Abe A., Kobayashi Y., Yamada G., 1998a, Analysis of subharmonic resonance of moderately thick antisymmetric angle-ply laminated plates by using method of multiple scales, Journal of Sound and Vibration, 217, 467-484

4. Abe A., Kobayashi Y., Yamada G., 1998b, Internal resonance of rectangular laminated plates with degenerate modes, JSME International Journal, Series C, 41, 718-726

5. Abe A., Kobayashi Y., Yamada G., 1998c, Two-mode response of simply supported, rectangular laminated plates, International Journal of Non-Linear Mechanics, 33, 675-690

6. Abe A., Kobayashi Y., Yamada G., 2000, Non-linear vibration characteristics of clamped laminated shallow shells, Journal of Sound and Vibration, 234, 405-426

7. Hoseini S.H., Pirpodaghi T., Asghari M., Farrahi G.H., Ahmadian M.T., 2008, Nonlinear free vibration of conservative oscillators with inertia and static type cubic nonlinearities using homotopy analysis method, Journal of Sound and Vibration, 316, 263-273 
8. Liao S.J., 1992, Proposed homotopy analysis techniques for the solution of nonlinear problems, Ph.D. Dissertation, Shanghai Jiao Tong University, Shanghai

9. Liao S.J., 1995, An approximate solution technique which does not depend upon small parameters: a special example, International Journal of Non-Linear Mechanics, 30, 371-380

10. Liao S.J., 2003, Beyond Perturbation: Introduction to Homotopy Analysis Method, Chapman \& Hall/CRC Press, Boca Raton

11. Liao S.J., 2004, On the homotopy analysis method for nonlinear problems, Applied Mathematics and Computation, 147, 499-513

12. LiaO S.J., 2009, Notes on the homotopy analysis method: some definitions and theorems, Communications in Nonlinear Science and Simulation, 14, 983-997

13. Liao S.J., 2012, Homotopy Analysis Method in Nonlinear Differential Equations, Springer Education Press, Heidelberg

14. Liao S.J., Cheung A.T., 1998, Application of homotopy analysis method in nonlinear oscillations, Journal of Applied Mechanics, 65, 914-922

15. LiAO S.J., TAN Y., 2007, A general approach to obtain series solutions of nonlinear differential equations, Studies in Applied Mathematics, 119, 297-355

16. Mastroberardino A., 2011, Homotopy analysis method applied to electrohydrodynamic flow, Communications in Nonlinear Science and Numerical Simulation, 16, 2730-2736

17. Mehrizi A.A., Vazifeshenas Y., Domairry G., 2012, New analysis of natural convection boundary layer flow on a horizontal plate with variable wall temperature, Journal of Theoretical and Applied Mechanics, 50, 4, 1001-1010

18. Mohammadpour A., Rokni E., Fooladi M., Kimiaeifar A., 2012, Bernoulli-Euler beams under different boundary conditions with non-linear Winkler type foundation, Journal of Theoretical and Applied Mechanics, 50, 2, 339-355

19. Mustafa M., Hayat T., Hendi A.A., 2012, Influence of melting heat transfer in the stagnationpoint flow of a Jeffrey fluid in the presence of viscous dissipation, Journal of Applied Mechanics, 79, 2, 4501-4505

20. NAyfeh A.H., Mook D.T., 1979, Nonlinear Oscillations, Wiley, New York

21. Pirbodaghi T., Ahmadian M.T., Fesanghary M., 2009, On the homotopy analysis method for non-linear vibration of beams, Mechanics Research Communications, 36, 143-148

22. Ray S.S., Sahoo S., 2015, Traveling wave solutions to Riesz time-fractional Camassa-Holm equation in modeling for shallow-water waves, Journal of Computational and Nonlinear Dynamics, 10, 6, 1026-1030

23. Sedighi H.M., Shirazi K.H., ZARE J., 2012, An analytic solution of transversal oscillation of quintic non-linear beam with homotopy analysis method, International Journal of Non-Linear Mechanics, 47, 777-784

24. Wen J., CAO Z., 2007, Sub-harmonic resonances of nonlinear oscillations with parametric excitation by means of the homotopy analysis method, Physics Letters A, 371, 427-431 